\title{
COMENTARIOS EN TORNO AL FERROCARRIL Y EL CRECIMIENTO ECONOMICO ESPAÑOL ENTRE 1855 Y 1913
}

\author{
FRANCISCO COMIN \\ Universidad de Valladolid
}

Qué duda cabe que el ferrocarril es un vehículo privilegiado para explorar la situación de la economía de un país, sobre todo en el momento en que se construye el grueso de las redes. Si uno no se limita a realizar una historia interna, técnica, jurídica o económica, no tiene más remedio que analizar las conexiones que se establecen entre ese moderno medio de transporte y el resto de la economía; esas relaciones son múltiples y ricas en significación para la historia económica de un país. Precisamente, en el libro objeto de comentario (1), esas relaciones son la principal preocupación del autor.

El ferrocarril era, en la segunda mitad del siglo pasado, el subsector económico que más relación tenía con el resto de la economía. Como demandante necesitaba una gran cantidad de factores de producción para su construcción, mantenimiento y explotación; como oferente proporcionaba unos servicios de difícil sustitución, y creaba economías externas no despreciables. Entre los factores de producción demandados por el ferrocarril hay que destacar las grandes sumas de capital necesarias para su puesta en funcionamiento, así como las materias primas y productos elaborados. Por otro lado, los servicios suministrados por el ferrocarril permitieron a muchos productores colocar sus productos en mercados más distantes y ampliar su volumen de ventas, limitado hasta entonces por los deficientes sistemas de transporte previos, a la par que el surgimiento de nuevas actividades. La aparición del ferrocarril produjo trastornos en los principales sectores de la economía; lo que, junto al volumen de sus necesidades, y al largo período de amortización de sus inversiones, condujo normalmente a la intervención del Estado. La forma concreta en la que el Estado promocionó el ferrocarril condicionó unos efectos de la construcción y explotación del mismo sobre la economía que fueron distintos de los que previsiblemente hubiesen ocurrido en el caso de que la ayuda del Estado se hubiese dirigido a otras realizaciones.

Pues bien, Antonio Gómez Mendoza estudia todos esos posibles efectos económicos del ferrocarril en España entre 1855 y 1913. Cuando es posible

(1) Antonio Gómez Mendozs, Ferrocarriles y cambio económico en España, 1855-1913, Madrid, Alianza, 1982. 
lo hace de forma cuantitativa. Junto a la cuantificación de las ganancias que la construcción del ferrocarril generó en la renta nacional española, A. Gómez Mendoza trata de estimar las repercusiones que el ferrocarril tuvo sobre los sectores más directamente influidos por su aparición: las industrias siderúrgica y hullera, como suministradores de productos, y dos subsectores agrícolas (el triguero y el vinícola), como demandantes de sus servicios.

A. Gómez Mendoza no es el primer historiador de la economía española que se ha ocupado de esos problemas; la novedad del libro comentado radica en los métodos utilizados para tratarlos. De esa innovación se derivan otras, consistentes en el derribo, o matización, de opiniones existentes sobre esas materias. En este libro se utiliza la metodología propuesta por la Nueva Historia Económica, y se aplican recetas analíticas ya ensayadas en otros países. Admitiendo que esas técnicas presentan problemas, hay que reconocer que su aplicación práctica por el autor comentado al caso español presenta, al menos, dos ventajas. Primera: se explicitan las hipótesis ideales alternativas que sirven de parangón a los hechos realmente acaecidos. Segunda: las exigencias de cuantificación arrojan subproductos interesantes que de otra manera quizá ni se hubiesen planteado. La primera ventaja es obvia; pasa como en los juicios de valor en Economía: puesto que el economista no puede desprenderse de los juicios de valor en sus análisis, lo mejor es que los explicite. Trasladando el problema a la cuestión que nos ocupa: puesto que normalmente se utilizan hipótesis alternativas ideales o contrafactuales para valorar determinadas realizaciones en la historia económica española, lo mejor será explicitarlas; de esa forma será posible discernir la conveniencia de los juicios emitidos. La segunda ventaja deriva de que la necesidad de cuantificación del método utilizado por A. Gómez Mendoza, le conduce a estudiar aspectos inéditos de la vida económica: esas aportaciones, funcionales para el autor, acaban siendo, quizá, más relevantes que el objeto para el cual fueron creadas y utilizadas.

Esas ventajas dotan a las conclusiones del libro comentado de un carácter más riguroso y contrastable que las emitidas con anterioridad; lo cual no quiere decir que necesariamente sean más acertadas. Antonio Gómez Mendoza desarrolla la acción de su libro en un escenario muy transitado previamente; lo que convierte el intento en una aventura peligrosa. Cuando Antonio Gómez Mendoza entra en el tema, se dispone de hipótesis largamente debatidas y asentadas en la disciplina. Y él las pretende combatir con una audacia encomiable. Las hipótesis previas, debidas a G. Tortella y J. Nadal principalmente, se pueden amalgamar, a costa quizá de desvirtuarlas, en tres grupos, referentes a cada uno de los previsibles efectos de la construcción del ferrocarril; a) demanda de capital: los ferrocarriles absorbieron una parte importante del ahorro nacional que podría haber sido destinado a otras 
empresas económicas: inversión en la industria, o, sencillamente, en la construcción de otra red viaria con trazado y características distintas; $b$ ) demanda de materias primas o productos intermedios: la legislación de ferrocarriles permitió la importación de la mayor parte de los materiales necesarios para la construcción y explotación de la red, con lo que se perdió la oportunidad de crear una industria nacional suministradora de esos productos; el sector más perjudicado por esa legislación sería el siderúrgico, y c) oferta de servicios de transportes: la construcción del ferrocarril fue prematura, ya que no existía demanda de transporte suficiente; o fue hecha para favorecer la exportación de productos del país y no para fomentar el comercio interior. Casi ninguna de esas hipótesis es sostenida por la investigación de A. Gómez Mendoza, según su autor. De forma general, el autor del libro comentado desautoriza las opiniones previas condensándolas en una única proposición: la política de desarrollo adoptada en 1855 no fue acertada porque cercenó las posibilidades de crecimiento de la industria española. A. Gómez Mendoza combate esa afirmación desde un triple frente: 1) demostrando, a través del cálculo del ahorro social, que el retraso en la construcción del ferrocarril hubiese ocasionado altos costes para la economía española; 2) mostrando la imposibilidad de que la industria siderúrgica española hubiese suministrado el material suficiente, en el mismo tiempo y al mismo precio que la industria extranjera, y 3) estimando las mejoras que el ferrocarril introdujo en la comercialización, exterior e interior, de los productos agrarios más significativos. Esas demostraciones no son, a pesar del rigor teórico y documental que incorporan, concluyentes, y exigen algunos comentarios.

Tengo que aclarar con respecto a estos comentarios que se referirán al aspecto argumental exclusivamente; las pruebas históricas que, para apoyar sus afirmaciones, presenta A. Gómez Mendoza son incuestionables, por lo menos para mi limitado conocimiento de esas materias. La parte empírica del trabajo comentado no admite ninguna objeción: las pruebas y testimonios aportados están buscados con la paciencia y la eficacia de un historiador que domina el oficio. La impresionante erudición es uno de los principales logros del libro. Como se verá, los datos que utiliza, junto a sus razonamientos impecables, ha permitido a A. Gómez Mendoza poner en entredicho algunas estimaciones y proposiciones previas. Donde hay un margen a la especulación, en el que se van a situar esos comentarios, es en la metodología general utilizada. El hecho de que en el libro de A. Gómez Mendoza existan muchos raciocinios explicitados en todas sus alternativas lógicas, permite seguir sus razonamientos sin lagunas desde el principio hasta el final; además, siempre advierte de los peligros en que incurre al rechazar alguna de las alternativas por aceptar otra. Es de alabar la forma ęn que el libro comentado ha conseguido trocar una materia árida por sí, en un discurrir 
fluido de razonamientos, encadenados entre sí por implicaciones lógicas y por información de la realidad, que exigen del lector la alerta suficiente para seguir la pista y conocer si la argumentación discurre correctamente, o si descarrila, o si se desvía a una vía de servicio o muerta. La trabazón entre las alternativas lógicas y los cálculos basados en la realidad parece sencilla en el libro de A. Gómez Mendoza; y, sin embargo, elaborarla es realmente complicado. En este terreno de las divagaciones teóricas es donde me voy a atrever a lanzar algún dardo que quizá se estrellará contra la armadura protectora del libro comentado. El riesgo que asumo es grande: los superficiales impactos no dejarán de provocar la respuesta de A. Gómez Mendoza que, dada su mejor artillería, podrá asestar algún que otro cañonazo. Vengamos al tema.

Las demostraciones no pueden ser concluyentes porque están basadas en tal cantidad de supuestos, que sus resultados están sujetos a la admisión de éstos. Para poner de manifiesto esta apreciación será suficiente con pasar revista a la argumentación sintética que, en las conclusiones, el autor reseñado dedica a la refutación de la proposición general que se acaba de reproducir. A. Gómez Mendoza comenta que, tras la estimación del ahorro social, está en condiciones de afirmar que los costes de una política progre. sista, en 1855, distinta a la realizada y favorable a la inversión en actividades directamente productivas hubiesen sido elevados. No compara, sin embargo, esos costes con los generados por la política realmente seguida. Cuando se comente el cálculo del ahorro social se comprobará que en el mismo no se tiene en cuenta el coste de oportunidad de los recursos destinados al ferrocarril; nada se sabe, pues, del coste efectivo de la política ferroviaria seguida en 1855, y nada se puede seguir respecto a si el coste de la actuación alternativa hubiese sido mayor. Volveremos luego sobre esto.

Gómez Mendoza comienza diciendo que el modelo proteccionista alternativo hubiese retrasado las obras de construcción de la red, dado que la industria siderúrgica no hubiese podido ampliar su producción al ritmo exigido por el tendido. En esta consideración van implícitos dos supuestos que, aunque parezcan razonables, no es imprescindible admitir, dado el terreno hipotético en que nos movemos: 1) que la red tenía que construirse al mismo ritmo que el que efectivamente conoció en España, y 2) que la industria siderúrgica no hubiese podido seguir ese ritmo. Hay experiencias de otros países que mueven a pensar que pueden darse situaciones contrarias a esos supuestos. Admitido que el segundo supuesto no se cumpliese, A. Gómez Mendoza considera que la política proteccionista alternativa a la realizada en 1855 hubiese tenido unos efectos secundarios indeseables para la economía. Esos efectos podrían haberse realizado, pero no necesariamente. Se dice, en primer lugar, que los precios de los productos siderúrgicos hubiesen 
sido más elevados al producirse en el país, lo que hubiese repercutido sobre las tarifas. No hace falta acudir al argumento de la industria naciente. Una subvención del Estado hubiese arreglado el problema. Claro que la finan. ciación de la construcción hubiese tenido su coste para el Estado y, a su través, para la economía española. Pero ¿no hubiese sido mejor financiar la construcción que, como se hizo luego, financiar la explotación de las líneas? El desarrollo armónico de la economía hubiese impedido, quizá, que éstas funcionasen con pérdidas. $Y$ además si se da cabida a soluciones intermedias del tipo de que no toda la producción tenía que realizarse, por lo menos al principio, en el interior, esa subvención podría haberse financiado con los fondos recaudados, por ejemplo, a través de un arancel fiscal sobre los materiales ferroviarios importados. Se añade, en segundo lugar, que de haberse favorecido la implantación de una industria siderúrgica a mediados de siglo, su localización habría sido Asturias; como más tarde el lugar idóneo hubiese sido Vizcaya, esa implantación temprana hubiese sido un lastre para la industrialización española. Ese es efectivamente un inconveniente de los innovadores, que tienen que adaptarse a las condiciones cambiantes. Sin embargo, habría que medir los costes y beneficios antes de afirmar la inconveniencia de una decisión; además, si el mineral vasco se importaba de otros países, ¿por qué no podría importarse desde Asturias? De esa manera, quizá hubiesen podido complementarse las dos regiones. Por último, se argumenta que esa hipotética industria siderúrgica se hubiese encontrado en situaciones de exceso de capacidad, cuando la demanda ferroviaria comenzó a decrecer. Eso no tiene por qué ser un inconveniente: en un proceso de crecimiento como el que estamos imaginando, no tienen por qué crecer sólo el sector ferroviario y el siderúrgico, por lo que habría posibilidad de colocar el exceso de hierros en otros sectores interiores; en el peor de los casos, no hay que excluir la vía seguida por otros países: colocar el excedente en aquellos países más atrasados que tuviesen regímenes librecambistas.

Todas éstas son situaciones posibles que no hay que despreciar cuando se está valorando una determinada medida de política económica a través de los métodos del análisis de coste-beneficio; ya que, según indica el propio A. Gómez Mendoza, el cálculo del ahorro social no sería más que un ejercicio de análisis de coste-beneficio retrospectivo. No parece que esa identificación sea afortunada. En primer lugar, en el análisis coste-beneficio no se toman determinadas decisiones como dadas, solamente se introducen restricciones técnicas o sociales predeterminadas; en el ahorro social se supone que algunas decisiones son inalterables (la construcción del ferrocarril a un ritmo determinado sería una de las funciones objetivo en el análisis costebeneficio, y no un dato como en el ahorro social). En segundo lugar, el cálculo 
del ahorro social no se corresponde con el cálculo del coste-beneficio; como mucho puede asimilarse al cálculo del beneficio y ni aún eso.

Si he entendido bien, el ahorro social consiste en valorar la contribución de transporte ferroviario a la Renta Nacional mediante la diferencia del precio en el medio de transporte alternativo y el ferrocarril. Para calcular esa diferencia de coste de transportar un volumen determinado de productos se supone que el ferrocarril permanezca cerrado durante un ejercicio. Para la teoría económica más formalizada no es ningún problema la maleabilidad del capital, siendo posible transformar, en este caso, una locomotora en una gabarra. A. Gómez Mendoza podía haber echado mano de ese capital mecano o capital gelatina de la teoría del crecimiento económico para estudiar la utilización alternativa del capital invertido en ferrocarriles. No ocurre así porque los ferrocarriles permanecen sencillamente cerrados y no se permite, en la situación alternativa, que esos recursos sean utilizados para ser invertidos en otros menesteres, no necesariamente relacionados con el transporte. Así las cosas nada tiene de anómalo que la situación ideal sea menos eficiente, y que la puesta en funcionamiento del ferrocarril produzca un ahorro social. La fórmula de ahorro social acoge un supuesto fuerte: es preciso transferir factores de producción hacia el sector transporte desde el resto de la economía; lo que, obviamente, si no se alteran las técnicas, supondrá una disminución de la producción distinta del transporte, que, valorada adecuadamente, indica el ahorro social. Claro que lo que habria que desembolsar para cubrir los servicios proporcionados por el ferrocarril en el caso de que éste se cerrase, puede ser menor que lo que se obtendría de la venta del capital incorporado en el mismo. Esta es una diferencia importante entre el método del ahorro social y el análisis coste-beneficio: además de medir los beneficios proporcionados por el ferrocarril, habría que medir sus costes, privados y sociales; sólo en el caso de que aquéllos superasen a éstos un economista actual habría aconsejado a los políticos del bienio progresista llevar a cabo la política ferroviaria desarrollada. La disparidad de esos procedimientos de análisis queda aún más patente cuando se tiene en cuenta que en el análisis coste-beneficio se comparan distintos proyectos de inversión: sería absurdo plantearse los efectos de los mismos en un solo año; hay que calcular los costes y beneficios sociales durante la vida útil del capital instalado, o cuando menos en un plazo de tiempo adecuadamente dilatado. En el cálculo del ahorro social se toma en consideración un solo ejercicio. El cálculo del costebeneficio no habría separado los efectos de la construcción del ferrocarril sobre otros sectores de la producción, proveedores o clientes, como se hace en el libro comentado, donde los efectos del ferrocarril sobre las industrias siderúrgica o hullera, y sobre la agricultura, son analizados de forma independiente al cálculo del ahorro social. Tal y como calcula A. Gómez Mendo- 
za, el ahorro social le hubiera resultado una cifra muy superior si hubiese incluido esas ganancias dinámicas. Aún así, A. Gomez Mendoza obtiene unas estimaciones del ahorro social del ferrocarril en España, en relación con la renta nacional, que son muy superiores a otras calculadas para otros países, si se exceptúa el caso de México: mientras en otros países el porcentaje se sitúa entre el 4 y el 5, en España se halla entre el 10 y el 23. ¿Quiere decir algo esa comparación? Yo pienso que no gran cosa, por la sencilla razón de que los cálculos de esas cifras exigen tal cantidad de supuestos, tanto sobre las características de los mercados de productos y de factores, y sus curvas de demanda, como sobre tráfico a considerar y medios de transporte alternativos utilizables, que la mínima alteración de uno de ellos conduce a trastornos considerables en la cuantía del ahorro social estimado. La prueba de ello se encuentra en que A. Gómez Mendoza reestima el ahorro social considerando la existencia de paro estacional en la agricultura, y el valor de aquél en términos de la renta nacional disminuye al 6,6 por $100, \mathrm{o}$ en el supuesto más extremo al 1,5 por 100 .

$Y$, desde luego, no pienso que a partir de las comparaciones internacionales de la magnitud de las estimaciones del ahorro social se puedan extraer valoraciones sobre la indispensabilidad del ferrocarril en el crecimiento económico de una nación: ¿fue mayor la aportación del ferrocarril al crecimiento económico en España o México que en Inglaterra o Estados Unidos? Para responder a esta pregunta razonablemente hacen falta más elementos de juicio que el valor del ahorro social en un año. Para finalizar con el ahorro social, quiero manifestar que a través de ese concepto no se puede resolver adecuadamente la polémica en torno a la idoneidad de la política económica del bienio progresista para impulsar el crecimiento económico español en el siglo pasado. El punto de comparación del ferrocarril no pueden ser las carretas o los mulos; ninguna combinación de ambos medios de transporte constituía la «mejor alternativa histórica del ferrocarril». Si se quiere resolver la cuestión en términos de cálculos de costes y beneficios sociales habrá que volver a los planteamientos de Nadal o Tortella: la alternativa debería ser una forma diferente de ferrocarril, en cuanto a la construcción, origen de los materiales y ritmo de la misma, y forma de la red; o, por qué no, hallándose en el terreno de las alternativas hipotéticas, la inversión de los capitales asignados al ferrocarril en actividades directamente productivas. La consideración de esas alternativas globales no tiene por qué ser descartada con argumentaciones fácticas: desde luego es poco probable que los capitales extranjeros hubiesen acudido a este país a financiar una red ferroviaria con materiales producidos en el interior, a un ritmo distinto, con otra configuración y sin las facilidades dadas por los sucesivos Gobiernos españoles; pero no parece mucho más verosímil que, una vez construidos, los ferrocarriles 
se hubiesen cerrado y toda su carga hubiese sido transportada en mulas y carretas. Que no se hayan explicitado las hipótesis alternativas no quiere decir que no pueda hacerse. Cuento con los problemas de construir modelos lo suficientemente especificados que permitan evaluar los beneficios y los costes de las distintas alternativas hipotéticas existentes para la economía española a mediados del siglo XIX, pero eso no tiene que acobardar a los estudiosos del período.

La utilidad del planteamiento de A. Gómez Mendoza es, sin embargo, grande. La mayor parte del capítulo referente al ahorro social está ocupada por los cálculos de las variables que le permiten obtenerlo, que son: el volumen de todos los servicios proporcionados por los ferrocarriles españoles; el coste medio del transporte por ferrocarril y por una combinación de medios alternativos: canal, mar y carretera; y los costes ocultos de utilizar. esos medios alternativos de transporte: lentitud e irregularidad. Este es al aspecto más interesante, a la par que útil, del capítulo 3, para el historiador de la economía. La documentación utilizada es amplia y adecuada, y el tratamiento que recibe ejemplar: la erudición de las noticias, la laboriosidad de los cálculos y la sucesión impecable de las argumentaciones que los unen, al amparo de las necesidades derivadas del modelo seguido, son asombrosas. El conocimiento de la producción y costes de las empresas ferroviarias, la navegación terrestre y de cabotaje y el transporte carretero durante la segunda mitad del siglo xix cuentan a partir de la publicación de este libro con unas aportaciones valiosas e imprescindibles para aquéllos que quieran conocer o investigar estos temas. Tan importantes como el cálculo del ahorro social visible son los beneficios ocultos proporcionados por el ferrocarril; los transportes ferroviarios eran más baratos; de ahi que el ahorro social fuese positivo. El ferrocarril proporcionaba además un servicio más rápido, más regular y más seguro. Eso mejoró la eficacia de la economía española acortando los períodos de entrega de las mercancías y haciéndolos más regulares, lo que a su vez mejoró las condiciones de comercialización y permitió reducir la inversión en existencias de las compañías. Esas cuestiones están bien analizadas, y permiten a A. Gómez Mendoza cuantificar la mejora que experimenta el ahorro social si se añaden algunas de esas ganancias ocultas.

En los capítulos 4 y 5 se analizan las repercusiones que el tendido del ferrocarril tuvo sobre la producción de dos sectores proveedores del ferrocarril: el siderúrgico y el hullero. El planteamiento de los mismos contrasta con la metodología del ahorro social seguida en el previo; está dentro de la línea tradicional de análisis de estas cuestiones. En una evaluación de tipo global de la idoneidad de la política seguida por los Gobiernos progresistas del bienio, como la utilizada en el capítulo 3, no deberían haberse dejado fuera estas conexiones con los sectores suministradores. El hecho de que 
no todas las aportaciones del ferrocarril al crecimiento económico estén recogidas en el cálculo del ahorro social hace necesarios estos dos capítulos, así como el 5 .

En el capítulo 4, Antonio Gómez Mendoza examina una de las ideas centrales del denominado fracaso de la industrialización en España: la construcción del ferrocarril en la forma en que se hizo fue la ocasión perdida para la siderurgia española. Para el autor del libro comentado esa tesis, sostenida principalmente por el profesor J. Nadal, implica la utilización de proposiciones hipotéticas, o contrafactuales como le gusta decir a A. Gómez Mendoza. Efectivamente, adoptar la teoría de la oportunidad perdida significa suponer que si los ferrocarriles hubiesen comprado productos españoles, la siderurgia se habría desarrollado, y de su mano lo habría hecho el resto de la economía. A. Gómez Mendoza trata de descalificar esa tesis demostrando que esa alternativa no era factible: no era posible que la industria siderúrgica española hubiese suministrado los materiales que necesitaba el ferrocarril, ni al ritmo exigido, ni al mismo precio que fue proporcionado por las importaciones. En cualquier caso, los ejercicios que A. Gómez Mendoza realiza para mostrar que no hubo tal oportunidad perdida, porque la industria siderúrgica no hubiera podido reaccionar adecuadamente, son interesantes y rigurosos, y le sirven para desterrar algunos tópicos sobre la capacidad de demanda de los ferrocarriles y sobre los costes de explotación de las líneas. Las estimaciones realizadas han permitido cuantificar la demanda neta de productos siderúrgicos ejercida por los ferrocarriles, desglosada en sus dos componentes, según el origen interior o exterior de los suministros. Sólo se tiene en cuenta la demanda de carriles, quedando excluido el hierro incorporado en el material móvil. La innovación más destacable es la incorporación al análisis de la posible demanda del ferrocarril, de las ventas que éste realizaba de hierro; al ser oferente de hierros viejos a la propia industria siderúrgica, las necesidades netas de los ferrocarriles son menores que si solamente se considera la demanda bruta.

Para el análisis de las necesidades de hierro de las compañías ferroviarias, A. Gómez Mendoza estudia con detenimiento y precisión la cantidad de carriles necesarios para el tendido inicial, y la vida útil de aquéllos para evaluar las cantidades necesarias para las reposiciones, así como la política de renovaciones de las grandes empresas. Esas consideraciones le permiten estimar el volumen de hierro demandado y, de pasada, matizar algunas afirmaciones previas sobre la rapidez de sustitución de los carriles de hierro por los de acero que sobreestimaban la relevancia de aquélla. Las facilidades dadas por la ley de 1855 condujeron a que los pedidos de las compañías se orientasen al exterior: por una parte, la franquicia arancelaria permitía 
que los precios de los productos ingleses fuesen inferiores a los de los españoles: a esa ventaja habría que añadir, según el autor del libro comentado, las ventas a plazo de las compañías extranjeras, y la política de las compañías ferroviarias españolas de utilizar los mismos proveedores que sus casas matrices extranjeras. Hasta 1890 la mayor parte de los carriles procedían del exterior; en el libro comentado se estiman las importaciones entre 1873 y 1913 de forma adecuada: a las importaciones libres de derechos, A. Gómez Mendoza ha añadido los carriles que se introducían por las partidas del arancel general, concluyendo que las estimaciones anteriores estaban infravaloradas; concretamente, la de R. Anes es un 45 por 100 inferior a la de A. Gómez Mendoza, a lo largo del periodo 1873-1913. Obviamente, infravalorar las importaciones significa dar mayor importancia al consumo de hierros españoles. A partir de la década de 1890 el peso de las importaciones dentro del consumo total de carriles disminuye: eso fue debido a un proceso de sustitución de importaciones posibilitado por el arancel de 1891, la depreciación de la peseta e innovaciones técnicas que permitieron reducir el peso del combustible. Los ferrocarriles también fueron importantes como oferentes de carriles usados; la legislación de 1855 condicionó que una parte importante de los mismos se destinase al mercado exterior: entre 1873 y 1913 se exportó el 48 por 100 de la oferta nacional. La parte que quedó en el interior fue reutilizada en gran medida por las propias compañías, ya que su reventa para fines distintos devengaba derechos para la Hacienda. Así, pues, la legislación de 1855 privó a la siderurgia española de hierros viejos a un bajo coste. En resumidas cuentas, la demanda neta de los ferrocarriles españoles de productos siderúrgicos ascendió solamente al 41 por 100 de la demanda en términos brutos. Calcula A. Gómez Mendoza que en el caso de que la red hubiera sido tendida con carriles españoles, la industria siderúrgica nacional hubiese tenido que incrementar su producción al doble entre 1855 y 1872; eso hubiese significado tasas de crecimiento difícilmente alcanzables (del 4,2 por 100 anual); ese aumento sería aún mayor si se añadiese el hierro necesario para fabricar los accesorios de vía. Esa ampliación de capacidad hubiese acarreado problemas cuando cayó la demanda de carriles a partir de 1865. A. Gómez Mendoza calcula que en 1866, el exceso de capacidad de la industria siderúrgica hubiese ascendido al 73 por 100 . Por otro lado, los carriles de producción nacional hubiesen supuesto un coste para las compañías de 47 millones de pesetas entre 1855 y 1872 ; en el caso de Norte se habrían incrementado los gastos de primer establecimiento en un 32 por 100. En el período $1903-1913$ se produce el afianzamiento de la producción siderúrgica española; la polémica gira en torno al peso de la demanda ferroviaria en ese proceso. Según los cálculos de A. Gómez Mendoza, la demanda de tipo ferroviario en ese período representó el 11 por 100 
de la producción nacional; en los diez años anteriores el porcentaje había sido del 5,2 por 100, lo que indica que la demanda ferroviaria fue más importante para la industria del hierro. No está de acuerdo el autor de este libro con la opinión de J. Nadal de que esos datos confirman la tesis de la oportunidad perdida a mediados de siglo, cuando la demanda de carriles era más intensa. Su argumento se basa en que considera que la estimación de Nadal, de que la producción de carriles de las fábricas vizcaínas había absorbido la quinta parte de la producción de hierros y aceros entre 1904 y 1913 , está sobrevalorada: sus estimaciones muestran que la demanda neta de los ferrocarriles solamente habría absorbido el 7,5 por 100 de la producción de acero en el período 1902-1913. Sigo opinando que mientras no se evalúen las hipótesis alternativas propuestas por Nadal y otros historiadores de la economía - unas fácticas y otras hipotéticas-, la pretensión de J. Nadal sigue siendo válida; incluso en el caso de que la importancia relativa de la producción de carriles en la primera década del siglo $\mathrm{xx}$ sea dos veces $\mathrm{y}$ media inferior de lo aceptado por Nadal. Como A. Gómez Mendoza reconoce, en 1902 la demanda de carriles era menos intensa que en 1855, y, sin duda alguna, la producción siderúrgica era muy superior a principios de siglo. La evolución dispar de esas dos variables indica que a mediados del siglo XIX la importancia relativa de la demanda ferroviaria dentro de la producción siderúrgica tenía que haber sido superior que a principios del siglo xx. Esa es la importancia que habría que medir para evaluar la posible aportación del ferrocarril al crecimiento económico. Que la demanda de carriles a mediados del siglo pasado era muy importante con respecto a la producción siderúrgica nacional queda patente en el capítulo que estamos comentando: en el período 1855-1872 los carriles importados consumidos superaban la producción agregada de todas las fábricas en el mismo período. En breve: no se puede decir en un mismo capítulo que la demanda de carriles era $\tan$ grande con respecto a la capacidad productiva instalada que hubiese sido imposible satisfacerla con la producción nacional, y que la demanda de carriles no era lo suficientemente importante con respecto a la produc. ción nacional como para ocasionar grandes aumentos de la producción. Aun contando con que la estimación de Nadal está sesgada al alza, su prueba a fortiori sigue siendo válida.

El capítulo 5 sigue ocupándose de los efectos de la demanda del ferrocarril sobre la producción nacional, en este caso de la industria hullera. En este capítulo se realiza una estimación del consumo de carbón por los ferrocarriles, tanto directo, para consumo de combustible por las locomotoras, como indirecto, utilizado por los hornos de fundición para obtener carriles. También se analiza la procedencia de los carbones según fuesen nacionales o extranjeros. La importancia del ferrocarril para la industria hullera fue gran- 
de. Aun contando con la mejor calidad de los carbones foráneos, la mayor parte de los carbones nacionales eran adecuados para el consumo de las locomotoras. La utilización del carbón foráneo dependía del coste de adquisición, siendo rentable en las zonas costeras, pero siendo desplazado por el carbón nacional en las zonas del interior. Las principales compañías intentaron aprovechar los recursos carboníferos de las comarcas atravesadas por sus líneas, rechazando la franquicia ofrecida por la ley de 1855, dado el mayor coste del carbón inglés en el interior. A. Gómez Mendoza estima que el combustible extranjero participó en una tercera parte en el consumo ferroviario en el período 1900-1913. La utilización del carbón español en la fabricación de carriles fue mucho menos importante que el consumo directo por los ferrocarriles: en primer lugar, porque hasta 1891 apenas se consumieron carriles nacionales; y, en segundo lugar, porque cuando creció la producción de carriles del país, la localización de la industria siderúrgica y la mayor calidad dc! carbón extranjero aconsejó importar el combustible. Incluso en la época en que las minas de hulla proporcionaron un mayor porcentaje del consumo de carbón siderúrgico, aquél solamente representaba el 0,7 por 100 de la producción agregada de 1903-1913. Por tanto, una primera conclusión de este capítulo es que el consumo directo de hulla por los ferrocarriles fue mucho más importante que el indirecto. Por otra parte, también se pone de manifiesto que los ferrocarriles españoles consumieron un porcentaje de la producción de carbón superior al consumido por cualquier otro sector: la cuarta parte de la producción de hulla fue consumida por el sector ferroviario. Esa demanda, por tanto, desempeñó un papel importante en la expansión de la producción hullera en la segunda mitad del siglo xIx; el desarrollo de ciertas cuencas mineras está muy relacionado con la marcha de la explotación ferroviaria. Las repercusiones del ferrocarril están claras, y el libro de A. Gómez Mendoza refuerza lo que en este sentido antes habian afirmado algunos autores, como Nadal. Sin embargo, el autor del libro comentado no puede dejar de señalar algunas inconveniencias que para la hipótesis de la oportunidad perdida se derivan del hecho de que, de haberse fomentado la industria siderúrgica nacional para abastecer a los ferrocarriles, aquélla se hubiera instalado con toda seguridad en Asturias; esos hornos hubiesen sido ineficaces tras la aceptación mundial del método de Bessemer.

Por fin, en el capítulo 6 se analizan las consecuencias del ferrocarril sobre la comercialización de algunos productos, agrícolas exclusivamente. Los sectores analizados son el triguero y el vinícola. No cabe duda tras el estudio de A. Gómez Mendoza, que el ferrocarril facilitó el comercio de granos al abaratar los costes de transporte; eso sirve tanto para el abastecimiento de la periferia con granos producidos en el interior, como para la introducción de trigos importados al interior: cuando en la década de los ochenta los 
trigos extranjeros invadieron las costas españolas, el ferrocarril se encargó de transportarlos al interior ocasionando la crisis agrícola y pecuaria, o por lo menos difundiéndola. Del análisis del transporte de granos por ferrocarril, en la década de los ochenta y entre 1901 y 1913, en relación con la renta nacional, y de la dirección y cuantía de los flujos comerciales se obtienen algunas conclusiones interesantes, que corroboran lo que hasta ahora se sabía sobre la formación de un mercado nacional de cereales y que confirman la importancia del ferrocarril para la economía cerealera, sobre todo en las épocas proteccionistas. Hacia 1913, concluye A. Gómez Mendoza, el ferrocarril comercializaba la mitad del trigo consumido en España y la tercera parte de las harinas, aunque esos porcentajes disminuían en épocas de crisis. La composición regional del comercio pone en evidencia los flujos de comercio de los cereales, siendo confirmado el hecho de que Castilla era la principal proveedora del mercado catalán, y el descenso de las importaciones de trigo por Santander desde el resto peninsular a partir de la pérdida de las últimas colonias en 1898. La relación de la crisis agrícola y pecuaria con el ferrocarril es examinada con precisión, y se establece que el origen de la crisis no puede imputarse al ferrocarril, así como tampoco el reforzamiento del proteccionismo a partir de 1891 . En resumidas cuentas, la actuación del ferrocarril consistió en consolidar las corrientes desde las zonas cerealeras del interior hacia los mercados consumidores de la periferia; a la acción del ferrocarril y a la política proteccionista hay que achacar la formación de un mercado nacional de productos cerealícolas a partir de 1890 , como pone de manifiesto la convergencia de los precios medios regionales hacia la media nacional.

Del análisis de las cantidades de vino transportadas por ferrocarril deduce A. Góomez Mendoza que los ferrocarriles ejercieron un efecto positivo sobre el mercado del vino, ya que las cantidades comercializadas por ferrocarril fueron en aumento desde mediados del siglo pasado. El ferrocarril no solamente acercaba los mercados al reducir el coste de transporte del vino, sino que al hacer accesibles los mercados exteriores posibilitó la exportación. Al reducir el tiempo y mejorar las condiciones de transporte se contribuyó a mejorar la calidad de los vinos. Por otro lado, se contribuyó a mejorar el abastecimiento de barricas de madera y a abaratar su coste. Utilizando el ahorro social, A. Gómez Mendoza llega a la conclusión de que ante un cierre de los ferrocarriles, las exportaciones de vinos ocasionadas por la filoxera en Francia hubiesen sido poco afectadas, ya que los viñedos estaban muy bien situados para esa exportación. El ferrocarril no solamente permitió enviar vinos al exterior, sino que también, y fundamentalmente, a partir de principios del siglo xx, contribuyó a la comercialización de los caldos en el mercado interior. La lectura de este capítulo es menos amena y atrayente que 
la de los anteriores, debido a la aridez del estudio de los flujos comerciales, a la menor utilización de la argumentación económica, tan visible en los capítulos previos, y a que sobre estas cuestiones de la formación del mercado interior no existen hipótesis arriesgadas y generalizadas como anteriormente se ha visto existían con respecto a los efectos de la política del bienio. Eso no quiere decir, sin embargo, que lo que se lee en este capítulo no sea importante; al contrario, abre una nueva perspectiva al estudio de la formación y configuración del mercado nacional de productos agrarios.

Es importante destacar que la argumentación de A. Gómez Mendoza, como él mismo apunta no contradice la noción de industrialización fallida en España entre mediados del siglo xIx y la Primera Guerra Mundial. Esa revolución de los transportes ocasionada por el tendido de las vías ferroviarias no fue acompañada de cambios en otros sectores, ni de una política de obras públicas complementarias del ferrocarril. Esa afirmación es importante y ya la habían acuñado otros historiadores, quienes, además, dieron alguna explicación a ese hecho. A. Gómez Mendoza ha estado tan preocupado por derribar algunas de esas explicaciones del fracaso de la industrialización en España, que no ha tenido oportunidad de ofrecer una propia y distinta. «Si los ferrocarriles fracasaron fue porque no se les secundó adecuadamente», afirma A. Gómez Mendoza. Esa es una explicación insuficiente; ¿hubo algún sector más secundado, ayudado o favorecido a mediados del siglo xIX que el ferroviario? La conclusión final del libro comentado es: «El nivel de desarrollo y el nivel de bienestar alcanzados por la economía española hubieran sido decididamente inferiores de no haberse tendido los ferrocarriles a partir de 1855." Pienso que no puede haber nadie que disienta de esa afirmación. La pregunta relevante a la que habrá que responder, aunque algunos ya lo han hecho, debería plantearse en los siguientes términos: ¿se habría alcanzado un nivel de desarrollo superior de haberse asignado los recursos disponibles por la economía española de otra manera? En el entendido - claro está- de que cerrar los ferrocarriles un año no es la manera más eficiente de reasignar esos recursos. Hay que continuar en la línea tradicional del análisis del fracaso de la industrialización en España; y por ese camino pueden llevarse la mayor parte de las aportaciones y precisiones del trabajo comentado, que no son pocas, así como algunas sugerencias metodológicas o de procedimiento: los cálculos impecables, los testimonios fidedignos, los supuestos y situaciones hipotéticas explicitadas, y los razonamientos ordenados y consistentes, son las principales cualidades del proceder de Antonio Gómez Mendoza en el libro comentado. A partir de la aparición de este libro debe replantearse gran parte de las tesis vigentes sobre la relación del ferrocarril con el crecimiento económico en la España de la segunda mi- 
tad del siglo xix. Creo que el trabajo de A. Gómez Mendoza no las derriba, aunque obligue a sus autores a afinarlas algo más.

Estas lucubraciones son los únicos reparos que con mi corto entendimiento en materias ferroviarias he podido objetar a este gran libro, cuyas principales aportaciones cuantitativas e históricas son de una gran valía. El hecho de que yo, como el propio A. Gómez Mendoza, conceda mayor importancia, y desde luego mucho más espacio en estos comentarios, a la crítica que a las lisonjas quizá haya conducido a formar una opinión sesgada a la baja de esta gran aportación a la historia de los ferrocarriles españoles en su relación con el cambio económico en la segunda mitad del siglo pasado. La lectura del libro, que recomiendo encarecidamente, sacará de ese malentendido. 